\title{
APLICAÇÃO DA ANÁLISE DE CONFIABILIDADE HUMANA A MANOBRAS DE NAVIOS EM PORTOS
}

\author{
Danilo Taverna Martins Pereira de Abreu \\ Universidade de São Paulo \\ Av. Prof. Mello Moraes, 2231, Cidade Universitária, São Paulo, SP \\ danilo.abreu@usp.br \\ Marcos Coelho Maturana \\ Universidade de São Paulo \\ Av. Prof. Mello Moraes, 2231, Cidade Universitária, São Paulo, SP \\ marcos@labrisco.usp.br \\ Marcelo Ramos Martins \\ Universidade de São Paulo \\ Av. Prof. Mello Moraes, 2231, Cidade Universitária, São Paulo, SP \\ mrmartin@usp.br \\ Siegberto Rodolfo Schenk Jr. \\ Conselho Nacional de Praticagem (CONAPRA) \\ Av. Rio Branco, 89 - 1502 - Centro, Rio de Janeiro, RJ \\ sieg.schenk@gmail.com
}

\begin{abstract}
RESUMO
Num contexto onde as dimensões dos navios são crescentes, as manobras em portos tendem a se tornar cada vez mais arriscadas, em função de resultarem em margens de segurança mais estreitas. Em função disso, torna-se necessária a melhoria contínua do desempenho dos operadores a bordo a fim de garantir a realização da manobra sem incidentes ou acidentes. Com o intuito de analisar quais são os principais fatores que influenciam no desempenho humano nas manobras em portos e, com isso, dar subsídios à tomada de decisão em ações de melhoria, este artigo apresenta os avanços na aplicação da análise de confiabilidade humana $(\mathrm{ACH})$ por meio de redes Bayesianas às manobras de navios em portos. Uma vez que a maioria dos trabalhos semelhantes se concentra em torno da navegação em mar aberto, uma das principais contribuições deste trabalho diz respeito à aplicação da $\mathrm{ACH}$ focada nos acessos portuários.
\end{abstract}

Palavra-chave: confiabilidade humana; redes Bayesianas; praticagem; análise de risco; navegação em águas restritas.

\begin{abstract}
In a context where ship sizes are increasing, maneuvering in ports tends to become increasingly risky due to the reduced safety margins. As a result, the continuous improvement of the operators' performance on board is necessary to ensure that the maneuver is carried out without incident or accident. In order to analyze what are the main factors that influence the human performance in port maneuvers and, thus, to support decision making regarding improvement actions, this article presents the advances in the application of human reliability analysis (HRA) through Bayesian networks to maneuvering ships in ports. Since most of the similar work focuses on open sea navigation, one of the main contributions of this work refers to the application of HRA focused on navigation in restricted waters.
\end{abstract}

Keywords: human reliability; Bayesian networks; pilotage; risk analysis; restricted waters navigation. 


\section{Como Citar:}

ABREU, D. T. M. P.; MATURANA, M. C.; MARTINS, M. R.; SCHENK JR., S. R. Aplicação da análise de confiabilidade humana a manobras de navios em portos. In: SIMPÓSIO DE PESQUISA OPERACIONAL E LOGÍSTICA DA MARINHA, 19., 2019, Rio de Janeiro, RJ. Anais [...]. Rio de Janeiro: Centro de Análises de Sistemas Navais, 2019.

\section{INTRODUÇÃ̃O}

Explicita-se que este artigo sintetiza, adapta e traduz conteúdo apresentado previamente na referência [1].

Em diferentes áreas industriais, existe o consenso de que o fator humano é um dos maiores contribuintes para a ocorrência de acidentes. $\mathrm{Na}$ indústria marítima essa tendência também é observada, dado que cerca de $80 \%$ ou mais de seus acidentes são causados de algumamaneira pelo erro humano [2]. Em resposta, a InternationalMaritimeOrganization (IMO) propõe em seu guia de análise de segurança (Guidelines for Formal Safety Assessment, FSA) que a análise de confiabilidade humana $(\mathrm{ACH})$ seja incluída nas análises probabilísticas de risco [3]. Em função disso, diferentes métodos de $\mathrm{ACH}$ foram propostos ao longo das últimas duas décadas, em diferentes áreas da indústria marítima.

Do conjunto de trabalhos focados especificamente na navegação, a grande maioria aborda o impacto do fator humano na navegação em mar aberto. Por exemplo, Martins e Maturana [4] apresentaram uma análise quantitativa da contribuição do erro humano em cenários de encalhe e abalroamento de navios petroleiros. Este trabalho modela a sequência de acidente por meio de uma árvore de falhas, na qual os eventos básicos são constituídos por erros humanos que, por sua vez, têm suas probabilidades estabelecidas por meio da THERP (Technique for HumanError Rate Prediction) [5]. Apesar de permitir a quantificação da probabilidade de acidente considerando a possibilidade de erro humano, essa abordagem é limitada em termos da representação de dependências e identificação dos fatores que influenciam o desempenho humano (Performance ShapingFactors, PSFs).

Um avanço na direção de superar ambas as limitações citadas anteriormente diz respeito à modelagem para a $\mathrm{ACH}$ por meio de redes Bayesianas (RBs), as quais permitem mapear relações causais entre fatores internos e externos ao operador e seu impacto no desempenho. $\mathrm{O}$ uso das RBs com esse fim se popularizou na comunidade de análise de risco devido à sua capacidade superior de modelagem em relação às técnicas de gerações anteriores. Nessa linha, na área de navegação marítima, autores como Martins e Maturana [6], Sotiralis et al. [7] e Hänninen e Kujala [8] modelaram o desempenho humano com o auxílio deRBs, o que permitiu a avaliação da contribuição dos PSFs à probabilidade de acidente.

Apesar do notável foco na navegação em mar aberto, a navegação em acessos portuários é uma das etapas mais críticas em termos de risco e, inclusive, vulnerabilidade a erros humanos. Diversos fatores implicam (ou podem implicar) em maior dificuldade na manobra, por exemplo: a) dimensões reduzidas (largura e profundidade do canal); b) o efeito squat; c) densidade do tráfego; d) velocidades menores, que implicam em redução da eficiência do leme; e) alterações na batimetria. Adicionalmente, diferentemente da navegação em mar aberto, os sistemas de piloto automático dos navios são desligados, o que naturalmente torna maior a necessidade de intervenção humana. Dadas esses desafios, em regiões portuárias, os navios são podem ser sujeitos à praticagem, atividade na qual o prático (piloto com experiência local de manobra) vai a bordo do navio para auxiliar o comandante na manobra e coordenar os rebocadores.

Num contexto onde as dimensões dos navios são crescentes [9], as manobras em portos tendem a se tornar cada vez mais arriscadas, em função de resultarem em margens de segurança mais estreitas. Em função disso, torna-se necessária a melhoria contínua do desempenho dos operadores a bordo a fim de garantir a realização da manobra sem incidentes ou acidentes. Com o intuito de analisar quais são os principais PSFs que influenciam no desempenho humano nas manobras em portos e, com isso, dar subsídios à tomada de decisão em ações de melhoria, este artigo apresenta os avanços na aplicação da $\mathrm{ACH}$ por meio de RBs às manobras de navios em portos. Uma vez que a maioria dos 
trabalhos semelhantes se concentra em torno da navegação em mar aberto, uma das principais contribuições deste trabalho diz respeito à aplicação da $\mathrm{ACH}$ focada nos acessos portuários.

\section{REDES BAYESIANAS}

As RBs são grafos acíclicos direcionados, nos quais os nós representam proposições (ou variáveis), os arcos representam as dependências diretas entre as proposições conectadas e a intensidade dessas dependências é dada por tabelas de probabilidades condicionais (TPCs) [10]. As RBs constituem estruturas de dados desenvolvidas para mapear dependências entre variáveis aleatórias. Esse tipo de estrutura permite modelar o processo de inferência do raciocínio humano, o qual pode ser subjetivo, incerto e incompleto.

A Figura 1 apresenta um exemplo de RB e explicita suas TPCs. Nessa rede, há três nós - A, B e C -, cada qual com dois estados possíveis, enumerados com os algarismos 1 e 2 . Os arcos indicam as relações entre os nós. De acordo com a direção dos arcos, os nós $\mathrm{A}$ e $\mathrm{C}$ são ditos nós pais de $\mathrm{B}$ e, de maneira inversa, $B$ é dito nó filho de A e C. Para os nós que não possuem pais (A e C), devem ser atribuídas probabilidades a priori para seus estados. Já para o nó que possui pais, deve ser atribuída uma TPC, que indica a probabilidade de cada um de seus estados, dadas as combinações de estados dos nós pais.

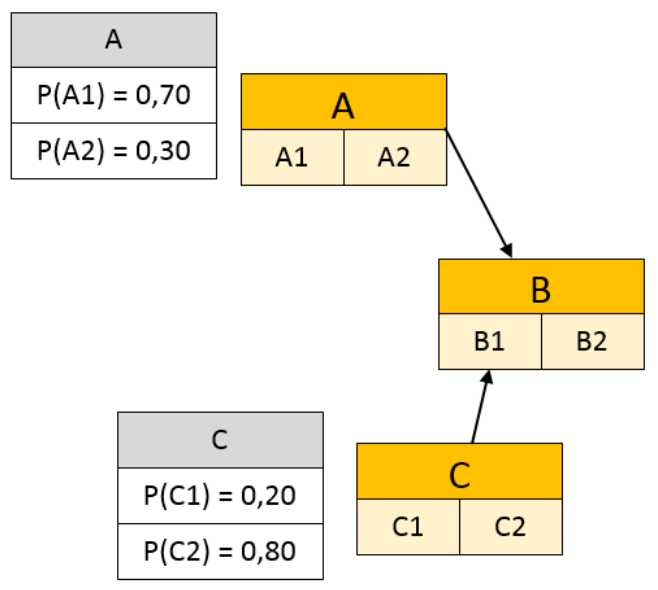

\begin{tabular}{|c|c|c|c|}
\hline$P(B 1)$ & $P(B 2)$ & $A$ & $C$ \\
\hline 0,30 & 0,70 & $A 1$ & $C 1$ \\
\hline 0,60 & 0,40 & $A 1$ & $C 2$ \\
\hline 0,40 & 0,60 & $A 2$ & $C 1$ \\
\hline 0,90 & 0,10 & $A 2$ & $C 2$ \\
\hline
\end{tabular}

Figura 1 - Exemplo didático de rede Bayesiana

Em suma, a especificação completa de uma RB deve atender as seguintes proposições [11]:

a) cada nó representa uma variável aleatória, que pode ser discreta ou contínua;

b) o conjunto de arcos direcionados representa as conexões entre pares de nós;

c) se há um arco que conecta o nó $X$ ao nó $Y$, então se diz que $X$ é o nó pai de $Y$ e cada nó $X_{i}$ possui uma distribuição de probabilidades condicionais $P\left(X_{i} \mid \operatorname{Pais}\left(X_{i}\right)\right)$ que quantifica o efeito dos nós pais no nó filho;

d) o grafo é direcional e acíclico.

As RBs apresentam um conjunto de possibilidades que favorecem a sua aplicação em diferentes áreas do conhecimento, tais como a inteligência artificial e, em especial, a análise de risco. Nessa última área, destacam-se as capacidades de realização de diagnósticos e prognósticos, o apelo visual da representação gráfica e a geração de resultados quantitativos.

\section{ACH POR MEIO DE REDES BAYESIANAS}

Os fatores que influenciam o desempenho humano na realização de uma tarefa se relacionam de maneira complexa. Dada a necessidade de representar essas relações para a modelagem computacional, Martins e Maturana [6] propuseram um modelo genérico de dependência, apresentado na Figura 2. 


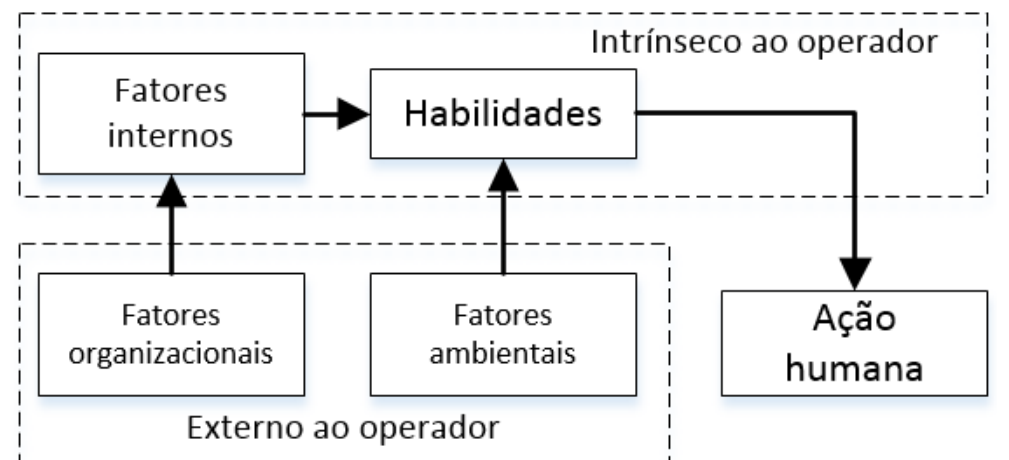

Figura 2 - Modelo genérico de dependência

O modelo genérico de dependência delibera que os erros humanos se originam a partir das ações necessárias para cumprir uma tarefa. O desempenho nessas ações, por sua vez, depende das habilidades, tais como concentração, percepção e comunicação, que são intrínsecas ao operador. As habilidades são influenciadas por fatores internos ao operador, como fadiga e qualidade do sono, além de fatores ambientais, como temperatura e visibilidade. Por fim, os fatores internos são influenciados pelos fatores organizacionais, como carga de trabalho e treinamento. No modelo em questão, não é considerada, por exemplo, a influência dos fatores organizacionais nas habilidades, dado o intervalo de tempo relativamente curto da operação.

As relações de dependência do modelo genérico podem ser mapeadas em uma RB, na qual cada nó representa um fator (interno, ambiental ou organizacional), habilidade ou ação humana.Os arcos da rede devem respeitar as relações estabelecidas no modelo genérico. Por exemplo, um nó de fator interno só pode ter como pais os nós dos fatores organizacionais.A intensidade da influência entre os nós será quantificada por meio dos valores introduzidos nas TPCs após sua quantificação - vide seção 4. A estrutura resultante está representada esquematicamente na Figura 3.

De acordo com a metodologia proposta por Martins e Maturana [6], a qual incorpora o modelo genérico de dependência (vide Figura 2), a implementação da $\mathrm{ACH}$ por meio de RBs passa por quatro estágios, a saber:

1) familiarização, na qual os analistas coletam informações a respeito da operação analisada e estudam a literatura relevante, procedimentos de trabalho e normas;

2) análise qualitativa, na qual os analistas descrevem as principais ações humanas desempenhadas, definem a relação de fatores necessários para a boa execução das ações (PSFs) e determinam a topologia da rede;

3) análise quantitativa, na qual os analistas preenchem as TPCs e quantificam a probabilidade de erro humano; e

4) incorporação, na qual os analistas implementam os resultados na análise de risco da operação analisada, bem como realizam uma análise de sensibilidade aos PSFs. 


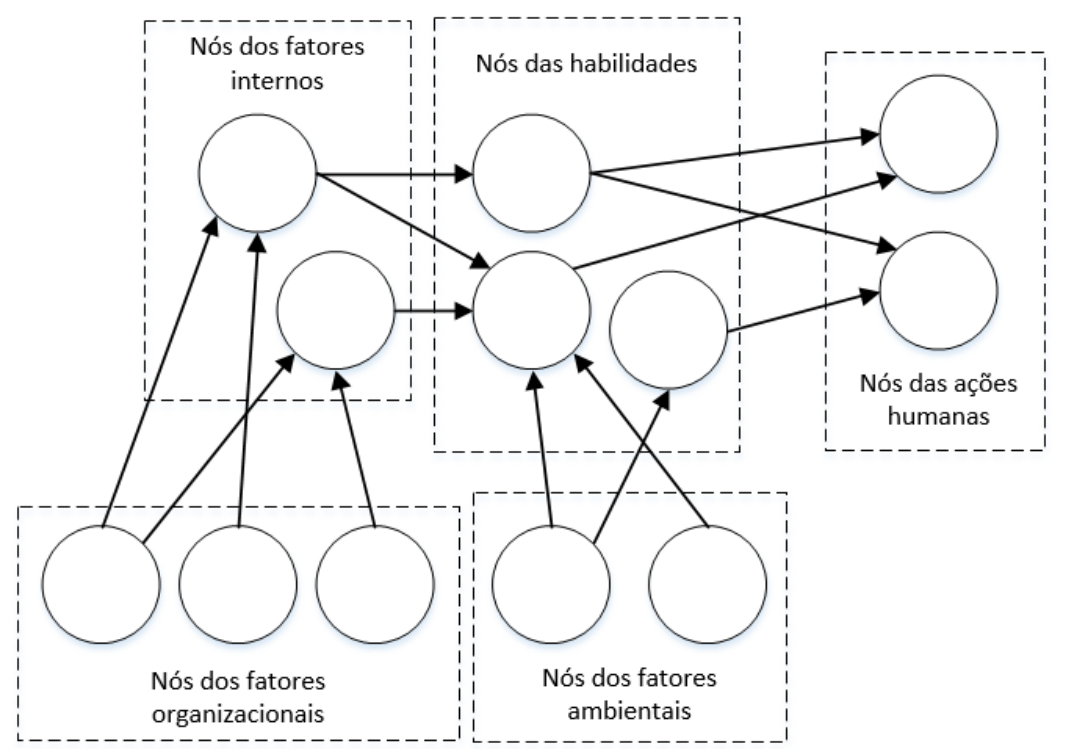

Figura 3 - Topologia da rede Bayesiana combinada com o modelo genérico de dependência

\section{QUANTIFICAÇÃO DA REDE BAYESIANA DE DESEMPENHO HUMANO}

No campo da $\mathrm{ACH}$, uma abordagem comum para a quantificação da $\mathrm{RB}$ diz respeito à consulta a especialistas (elicitação) da área estudada, devido à possível indisponibilidade de dados históricos para as relações complexas previstas na estrutura da rede. À medida que a rede aumenta de tamanho - i.e., são incluídos novos nós e arcos - a complexidade do processo de elicitação também aumenta. Quando isso ocorre, propõe-se na literatura que sejam quantificados junto a especialistas somente os valores extremos das TPCs (âncoras) e interpolados os demais. Como alternativa para preencher as TPCs sem depender da elicitação de especialistas, a qual pode rapidamente se tornar custosa e inviável, neste trabalho é proposto um método fundamentado em interpolações ponderadas pelas probabilidades de erro humano, previamente calculadas por meio de um modelo prospectivo de desempenho.

O modelo prospectivo em questão, desenvolvido por meio da TECHR (Technique for Early Consideration of Human Reliability), foi concebido por Maturana [12] com o propósito de permitir a análise quantitativa do desempenho humano nas fases de concepção de sistemas e aproveita dados de confiabilidade humana de diversas obras da literatura. Este modelo se baseia na taxonomia de Bloom [13] para as ações humanas e de Reason [14] para os tipos de erros humanos. Por meio da associação entre as probabilidades de erro humano e as ações humanas genéricas, o modelo estabelece distribuições de probabilidade para o erro em cada ação.

A Tabela 1 apresenta as probabilidades de erro humano para as ações humanas genéricas identificadas como necessárias ao cumprimento da tarefa analisada neste trabalho - vide seção 5 . As probabilidades de erro humano para outras ações genéricas podem ser consultadas em [12]. Na RB de desempenho humano os nós de ações humanas possuem apenas dois estados - "falha" e "sucesso" e as probabilidades de erro obtidas por meio da TECHR são colocadas como valores de referência para o estado de falha. Reforça-se que a soma entre a probabilidade de ambos os estados deve ser sempre unitária.

Tabela 1 - Probabilidade de erro para as ações humanas genéricas selecionadas

\begin{tabular}{cc}
\hline Ação humana genérica & Probabilidade de erro (média) \\
\hline Habilidade perceptiva & $3,69 \mathrm{E}-02$ \\
Interpretar & $4,28 \mathrm{E}-02$ \\
Checar & $1,57 \mathrm{E}-01$ \\
Planejar & $2,06 \mathrm{E}-01$ \\
Responder & $1,11 \mathrm{E}-01$ \\
\hline
\end{tabular}


Assim como os nós de ações humanas, os nós das demais categorias da RB possuem dois estados: um positivo e outro negativo. $\mathrm{O}$ estado positivo se refere à condição do fator ou habilidade que leva a uma melhoria de desempenho do operador, i.e., reduz a probabilidade de erro. $\mathrm{O}$ estado negativo se refere à condição oposta, i.e., aumenta a probabilidade de erro. A soma entre a probabilidade do estado positivo e a probabilidade do estado negativo de um nó deve ser sempre unitária. Feitas essas considerações, o procedimento de preenchimento quantitativo da rede deve seguir os seguintes passos:

- Passo 1: atribuir probabilidades a priori aos estados dos nós sem pais (fatores organizacionais e ambientais);

- Passo 2: fixar o valor dos âncoras para os nós de fatores internos e habilidades;

- Passo 3: interpolar os valores das TPCs dos nós de fatores internos e habilidades linearmente, com base nos valores dos âncoras;

- Passo 4: estabelecer valores arbitrários para os âncoras das TPCs dos nós de ações humanas, bem como as equações de interpolação das TPCs (os valores arbitrários serão modificados posteriormente);

- Passo 5: modificar os valores dos âncoras das TPCs dos nós de ações humanas, num processo de otimização ou similar, até que a diferença absoluta entre a probabilidade marginal do estado de falha do nó de ação humana e o seu valor correspondente na tabela de probabilidade de erro humano (Tabela 1) seja mínima.

No que diz respeito aos passos 2 e 3, devem ser estabelecidos para cada TPC (dos nós de fatores internos e habilidades) um valor mínimo, MIN, atribuído à probabilidade do nó filho estar no estado positivo, dado que todos os nós pais estão em seus estados negativos. Com isso, a probabilidade do nó filho estar no estado negativo será igual a 1 - MIN. De maneira similar, um valor máximo, MAX, deve ser atribuído à probabilidade do nó filho estar no estado positivo, dado que todos os nós pais estão em seus estados positivos. Naturalmente, a probabilidade do estado negativo deste nó filho deve ser igual a 1 - MAX. Então, a interpolação da tabela - i.e., a determinação da probabilidade de ocorrência dos estados dos nós filhos para outras combinações de estados dos nós pais - é feita com base no número de nós pais em seu estado positivo [6].

Sejam $P_{P O S}$ e $P_{N E G}$, respectivamente, as probabilidades dos estados positivo e negativo do nó filho; $n$ o número total de nós pais associados ao nó filho; e $n_{P}$ o número de nós pais em seu estado positivo para um estado específico. Então, as probabilidades dos estados dos nós filhos serão dadas matematicamente por:

$\mathrm{P}_{\mathrm{pOS}}=\mathrm{MIN}+(\mathrm{MAX}-\mathrm{MIN}) \cdot \mathrm{n}_{\mathrm{p}} / \mathrm{n}$

$\mathrm{P}_{\mathrm{NEG}}=1-\mathrm{P}_{\mathrm{pOS}}$

Na próxima seção, será apresentado um exemplo de preenchimento segundo este método.

Por sua vez, o equacionamento para preenchimento das TPCs dos nós de ações humanas segue exatamente a mesma regra de interpolação (passo 4). Entretanto, os valores de MIN e MAX devem ser modificados em um problema de otimização, a fim de minimizar o valor absoluto da diferença entre as probabilidades de falha na ação humana calculadas pela $\mathrm{RB}$ e as probabilidades de erro humano obtidas por meio da TECHR. Se $\mathrm{F}$ é a probabilidade de falha na ação humana calculada pela rede e PEH é a probabilidade de erro humano obtida pela TECHR, então o problema de otimização terá a seguinte forma:

$\operatorname{minimizar}(\mathrm{F}-\mathrm{PEH})^{2}$

sujeito a: $0 \leq$ MIN $<M A X \leq 1$

Essa metodologia de preenchimento provê algumas propriedades interessantes à RB de desempenho humano. Primeiramente, garante-se que os nós pais tenham influência cumulativa em relação aos nós filhos, ou seja, quanto mais fatores na rede estiverem em estados negativos, pior será o desempenho do operador (maior probabilidade de erro humano) e vice-versa. Além disso, a interface entre nós de 
habilidades e nós de ações humanas calibra a rede segundo as probabilidades obtidas da literatura e bancos de dados consagrados, o que compatibiliza os resultados da rede com aqueles que seriam obtidos por meio de outras metodologias.

\section{APLICAÇÃO A MANOBRAS DE NAVIOS EM PORTOS}

As manobras de navios em portos são tarefas sensíveis, altamente dependentes do desempenho dos operadores envolvidos. Comparadas à navegação em mar aberto, as dimensões navegáveis são significativamente menores e interações hidrodinâmicas com o fundo e com outros navios interferem no comportamento da embarcação manobrada. Não obstante, o projeto dos navios geralmente não é otimizado para estes trechos de navegação, o que incorre em desafios adicionais. Em função disso, torna-se interessante a compreensão de como os erros humanos ocorrem e os principais fatores que contribuem para isso, uma vez que essa informação contribui para a tomada de decisão em termos de melhorar a segurança do porto e, inclusive, sua eficiência.

Neste sentido, os benefícios de aplicar o modelo apresentado na seção anterior incluem não só a quantificação das probabilidades de erro humano, mas também a capacidade de verificar quais fatores mais impactam para essa probabilidade. Para ilustrar esse estudo, nessa seção é apresentado um exemplo simplificado de aplicação, voltado para a tarefa de manutenção do controle do navio pelo prático durante a navegação no canal.

Antes de construir a rede, os autores foram a bordo de navios durante suas manobras no Porto de Santos - SP, a fim de acompanhar a operação completa e identificar as principais tarefas desempenhadas pelos operadores. Ao todo, sete manobras foram observadas localmente para apoiar o estudo. Adicionalmente, os autores também acompanharam simulações no Tanque de Provas Numérico da Universidade de São Paulo, onde também tiveram a oportunidade de entrevistar os práticos. Ao todo, aproximadamente 110 horas de simulação foram acompanhadas. Esta atividade constituiu o primeiro estágio do trabalho, de familiarização, como previsto no procedimento descrito na seção 3 .

Em seguida, no segundo estágio do trabalho, foi feita a análise qualitativa. Os resultados da etapa de familiarização foram inicialmente registrados no formato de uma Análise Hierárquica de Tarefas (AHT), a qual decompõe uma tarefa em subtarefas até o limite de resolução desejado [15]. O nível de resolução adotado foi o de subtarefas que podem ser descritas em termos das ações humanas genéricas (AHG) previstas na taxonomia de Bloom. Para a tarefa analisada, a AHT está apresentada na Figura 4. A cada subtarefa identificada no nível de resolução adotado, foi atribuída uma das AHGs previstas na Tabela 1. 


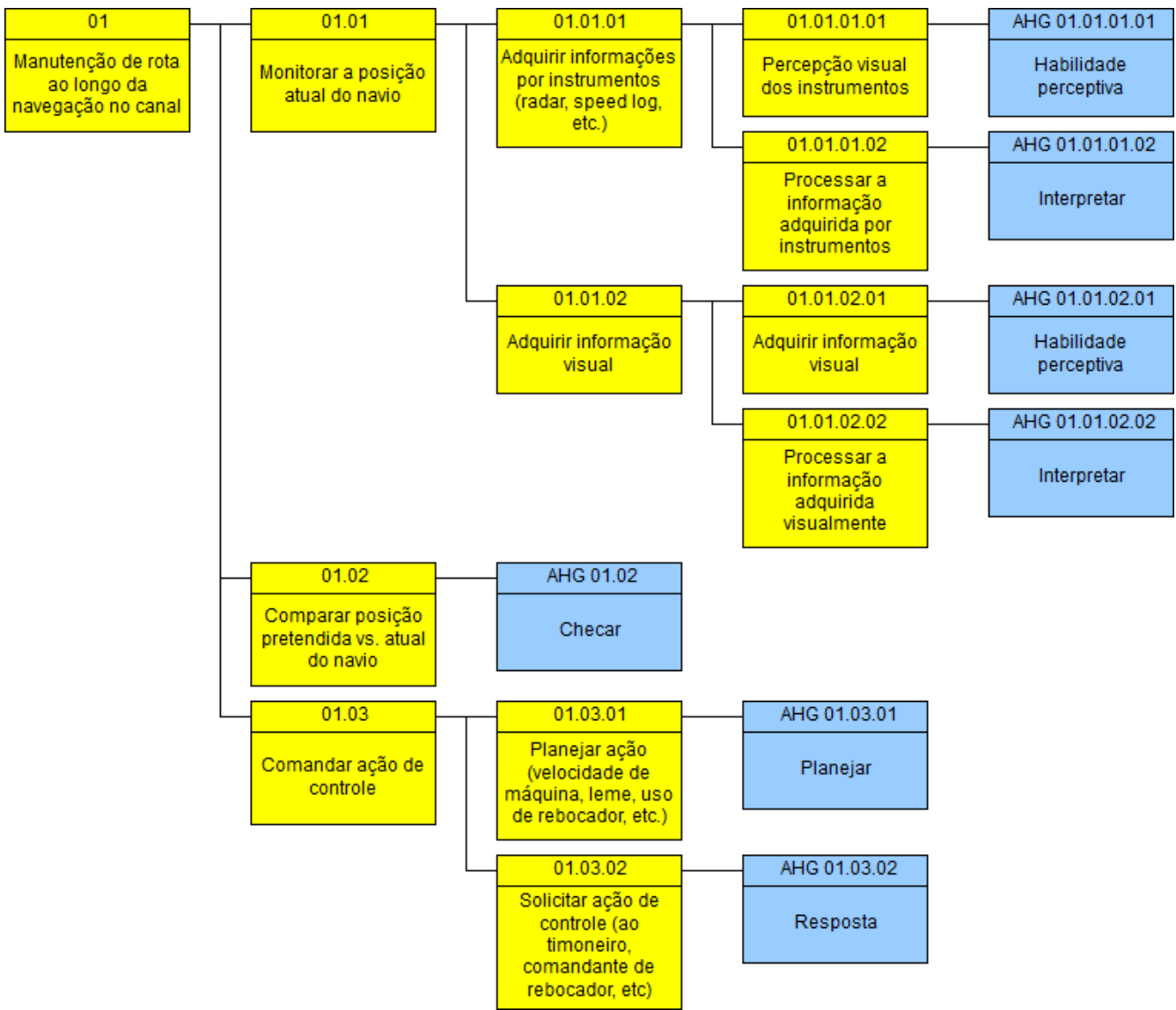

Figura 4 - AHT para a tarefa de manutenção da rota do navio ao longo da navegação no canal

As AHGs resultantes da AHT foram introduzidas como nós de ações humanas na RB. Após isso, foram determinadas as habilidades necessárias para a realização dessas tarefas. Em seguida, foram levantados os fatores internos e ambientais que impactam na tarefa, bem como os fatores organizacionais que modificam os fatores internos, como previsto no modelo genérico de dependência. Apenas as principais relações causais entre os fatores foram levantadas, para garantir a viabilidade computacional da rede.

A Tabela 2 apresenta todos os nós utilizados na RB, separados por categorias. Essa tabela também serve como um sumário dos fatores considerados na análise. Adicionalmente, a Figura 5 apresenta a estrutura completa da RB para a análise de desempenho humano e destaca as categorias às quais cada nó pertence. O software utilizado na modelagem foi o Netica 4.11. As relações de influência entre nós foram estabelecidas também durante a etapa de familiarização por meio de entrevistas aos especialistas.

No que diz respeito ao preenchimento quantitativo da $\mathrm{RB}$, foi seguido o procedimento descrito na seção 4 adotando os valores de 9,99E-01 e 1,00E-03, respectivamente, para as variáveis MAX e MIN das TPCs dos nós de habilidades e fatores internos. O preenchimento de uma TPC segundo o procedimento de interpolação está exemplificado na Tabela 3, para o nó "tratamento dado à falha".

Ainda, para os nós de fatores externos - ambientais e organizacionais -, foram atribuídas probabilidades a priori de 9,90E-01 para o estado positivo e 1,00E-02 para o estado negativo. Por fim, o equacionamento da rede foi reproduzido em uma planilha eletrônica e as TPCs dos nós de ações humanas foram obtidas por meio da resolução do problema de otimização mencionado anteriormente, resolvido no Solver do Microsoft Excel. 
Tabela 2 - Nós da RB e suas categorias

\begin{tabular}{|c|c|c|c|}
\hline Categoria & Nome do nó & Categoria & Nome do nó \\
\hline Habilidade & Conhecimento de procedimentos & Fator interno & Conhecimento das normas \\
\hline Habilidade & Percepcão & Fator organizacional & Carga de trabalho \\
\hline Habilidade & Liderança & Fator organizacional & Formalização \\
\hline Habilidade & Criatividade & Fator organizacional & Coordenação do trabalho \\
\hline Fator ambiental & Temperatura & Fator organizacional & Prêmios, recompensas e benefícios \\
\hline Fator ambiental & Barulho & Fator organizacional & Infraestrutura \\
\hline Fator interno & Fadiga & Fator organizacional & Qualidade de vida \\
\hline Fator interno & Dor e desconforto & Fator organizacional & Avaliação de performance \\
\hline Fator interno & Qualidade do sono & Fator organizacional & Programas da companhia \\
\hline Fator interno & Tratamento dado à falha & Fator organizacional & Seleção de recursos humanos \\
\hline Fator interno & $\begin{array}{l}\text { Comportamento baseado na influência do } \\
\text { grupo }\end{array}$ & Fator organizacional & Comunicação oral e escrita \\
\hline Fator interno & Identificação com a equipe & & \\
\hline
\end{tabular}

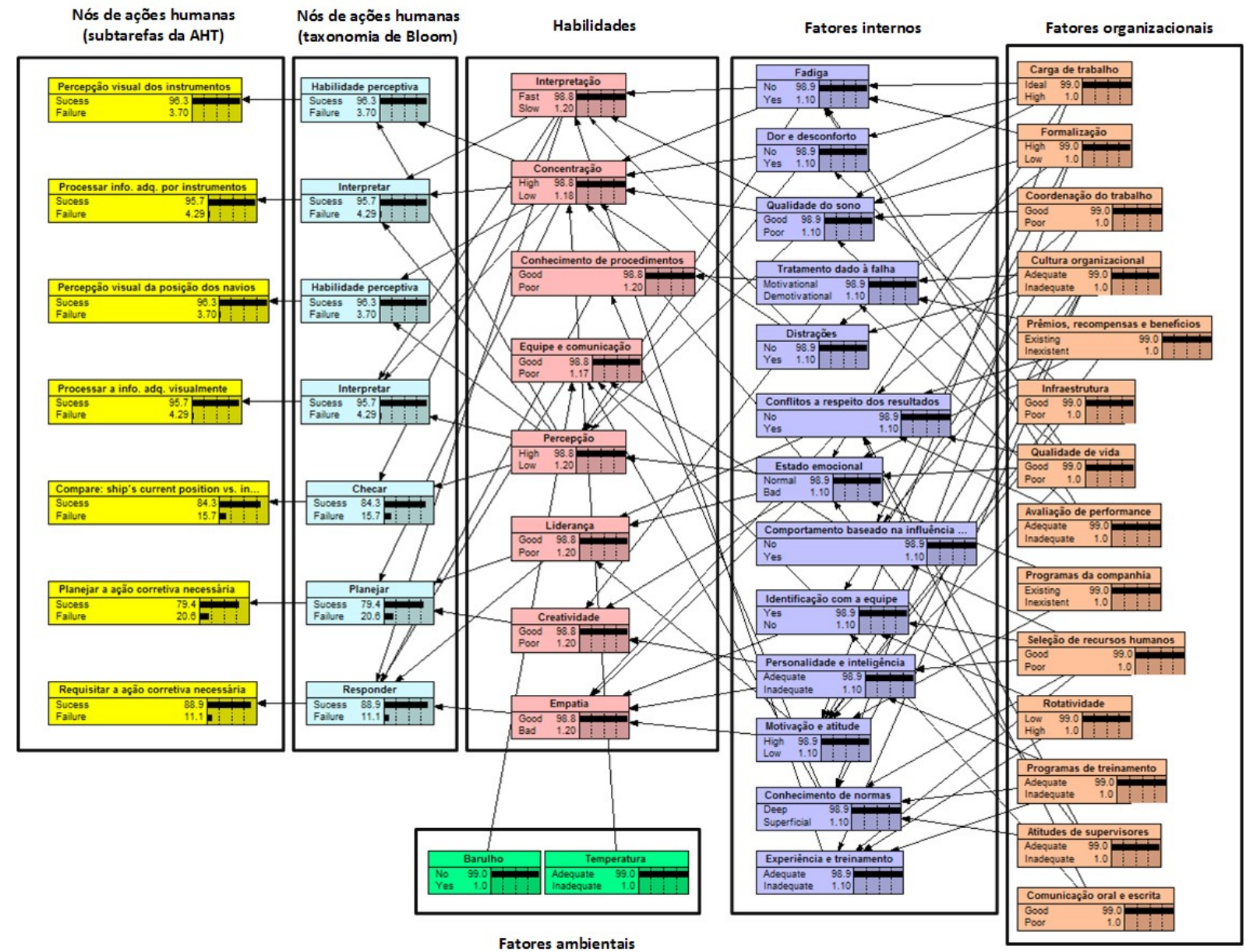

Figura 5 - RB para análise do desempenho humano na tarefa de manutenção da rota do navio 
Tabela 3 - Preenchimento da TPC do nó "tratamento dado à falha"

\begin{tabular}{cccccc}
\hline $\begin{array}{c}\text { Cultura orga- } \\
\text { nizacional }\end{array}$ & $\begin{array}{c}\text { Prêmios, re- } \\
\text { compensas e } \\
\text { benefícios }\end{array}$ & $\begin{array}{c}\text { Avaliação de } \\
\text { desempenho }\end{array}$ & $\begin{array}{c}\text { Número de } \\
\text { estados posi- } \\
\text { tivos }\end{array}$ & $\begin{array}{c}\text { Motivador } \\
\text { (estado positi- } \\
\text { vo) }\end{array}$ & $\begin{array}{c}\text { Desmotivador } \\
\text { (estado negati- } \\
\text { vo) }\end{array}$ \\
\hline Adequado & Existentes & Adequada & 3 & $9.99 \mathrm{E}-01$ & $1.00 \mathrm{E}-03$ \\
Adequado & Existentes & Inadequada & 2 & $6.66 \mathrm{E}-01$ & $3.34 \mathrm{E}-01$ \\
Adequado & Inexistentes & Adequada & 2 & $6.66 \mathrm{E}-01$ & $3.34 \mathrm{E}-01$ \\
Adequado & Inexistentes & Inadequada & 1 & $3.34 \mathrm{E}-01$ & $6.66 \mathrm{E}-01$ \\
Inadequado & Existentes & Adequada & 2 & $6.66 \mathrm{E}-01$ & $3.34 \mathrm{E}-01$ \\
Inadequado & Existentes & Inadequada & 1 & $3.34 \mathrm{E}-01$ & $6.66 \mathrm{E}-01$ \\
Inadequado & Inexistentes & Adequada & 1 & $3.34 \mathrm{E}-01$ & $6.66 \mathrm{E}-01$ \\
Inadequado & Inexistentes & Inadequada & 0 & $1.00 \mathrm{E}-03$ & $9.99 \mathrm{E}-01$ \\
\hline
\end{tabular}

Apesar de essas probabilidades não necessariamente refletirem uma operação em especial, esse preenchimento inicial já permite a realização de análise de sensibilidade, dado que essa depende em grande medida das relações causais de cada nó. Neste trabalho, a análise de sensibilidade foi realizada pelo modo de cálculo "entropy reduction" do software Netica. Dada uma variável de saída, $Q$, com $q$ estados, e uma variável de entrada, $F$, com $f$ estados, a redução de entropia (medida de sensibilidade), ${ }^{I}$, é calculada por [16]:

$I=H(Q)-H(Q \mid F)=\sum_{q} \sum_{f} \frac{P(q, f) \log _{2}[P(q, f)]}{P(q) P(f)}$

Onde $H(Q)$ é a entropia de $Q$ antes de quaisquer evidências na rede e $H(Q \mid F)$ é a entropia de $Q$ após as evidências da variável $F$, ambas medidas em bits de informação. Na prática, quanto maior a redução de entropia, mais uma variável de entrada influencia a variável de saída [17].

Os resultados obtidos da análise de sensibilidade para cada tarefa estão apresentados da Figura 6 à Figura 9. Esses resultados mostram que os fatores organizacionais "carga de trabalho" e "formalização" são os dois de maior impacto, uma vez que eles influenciam fatores internos como a fadiga e a qualidade do sono. Estes fatores internos, por sua vez, influenciam as habilidades de interpretação, concentração e percepção, as quais impactam diretamente nas capacidades cognitivas do operador. Adicionalmente, para tarefas interpessoais (e.g., requisitar ação corretiva), diferentes fatores organizacionais se mostram como os mais importantes, tais como "cultura organizacional" e "seleção de recursos humanos", devido à relação com fatores internos como "personalidade e inteligência" e "identificação com a equipe", os quais se relacionam com habilidades também interpessoais, como "empatia" e "liderança".

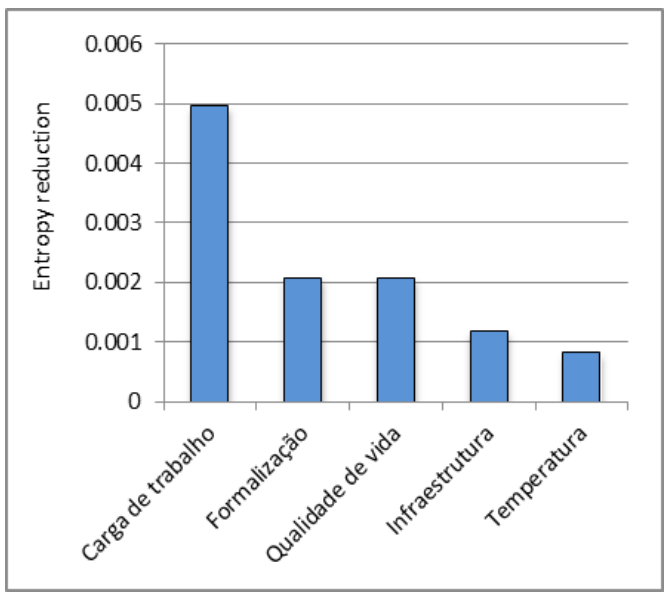

(a)

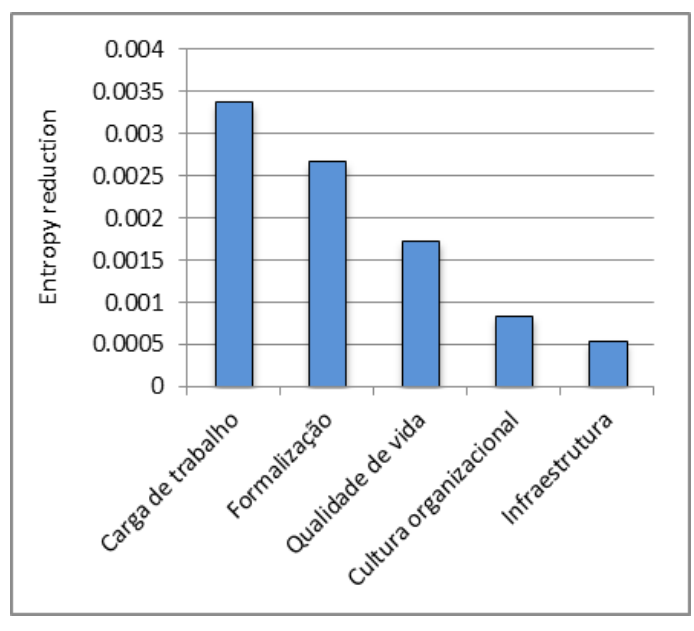

(b) 
Figura 6-Resultados da análise de sensibilidade para as subtarefas: (a) "percepção visual dos instrumentos"; (b) "processar informação adquirida por instrumentos"

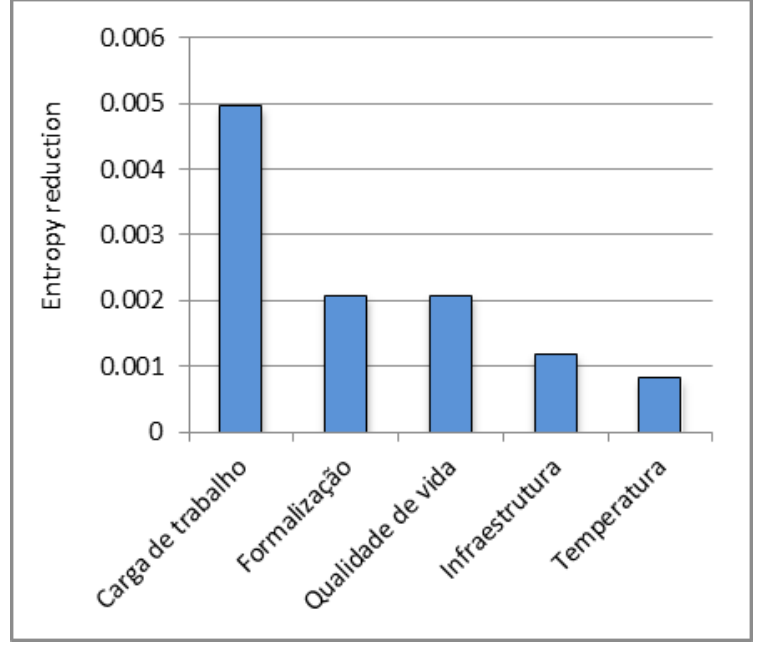

(a)

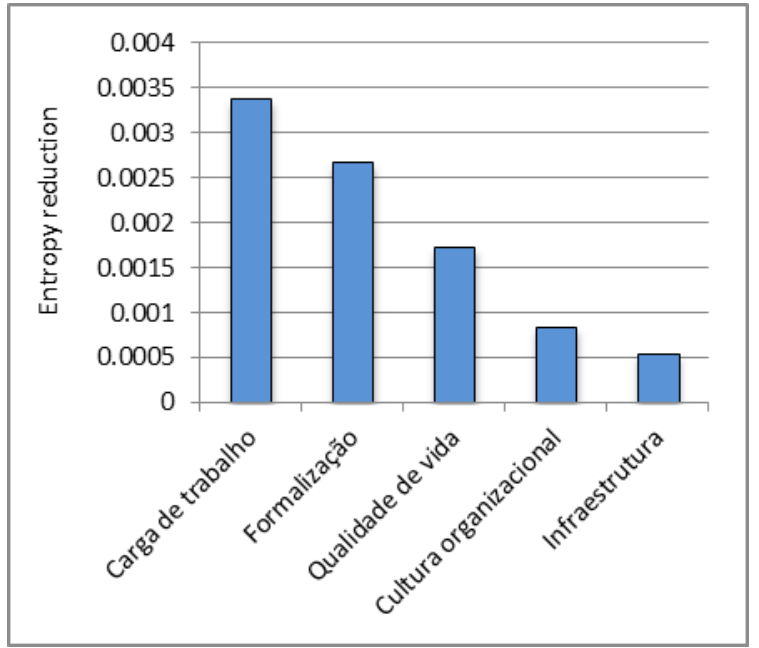

(b)

Figura 7-Resultados da análise de sensibilidade para as subtarefas: (a) "percepção visual da posição do navio"; (b) "processar a informação adquirida visualmente"

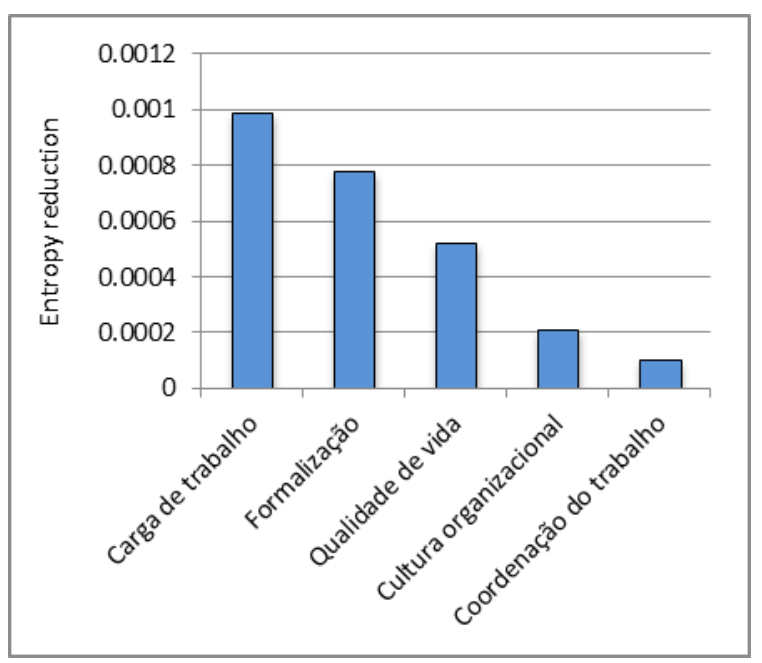

(a)

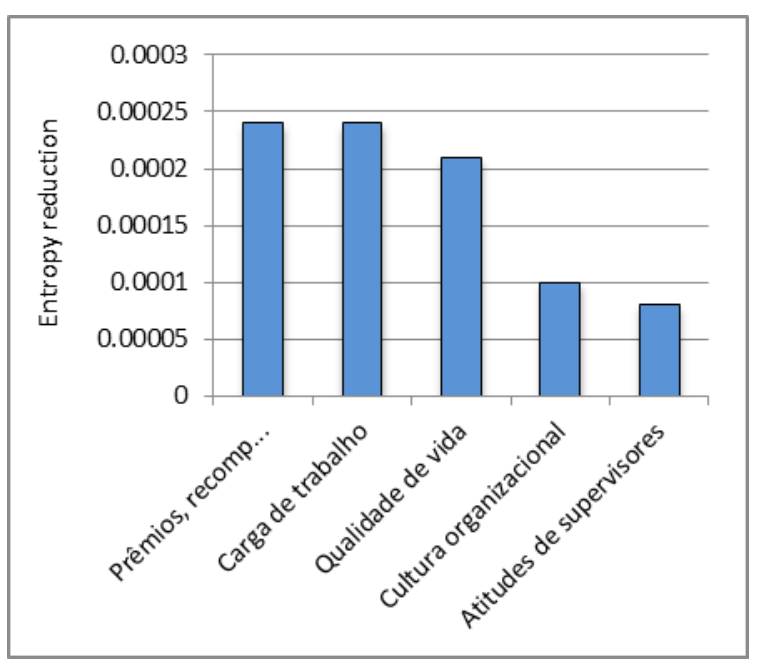

(b)

Figura 8-Resultados da análise de sensibilidade para as subtarefas: (a) "comparar a posição atual do navio vs. pretendida”; (b) "planejar a ação corretiva necessária" 


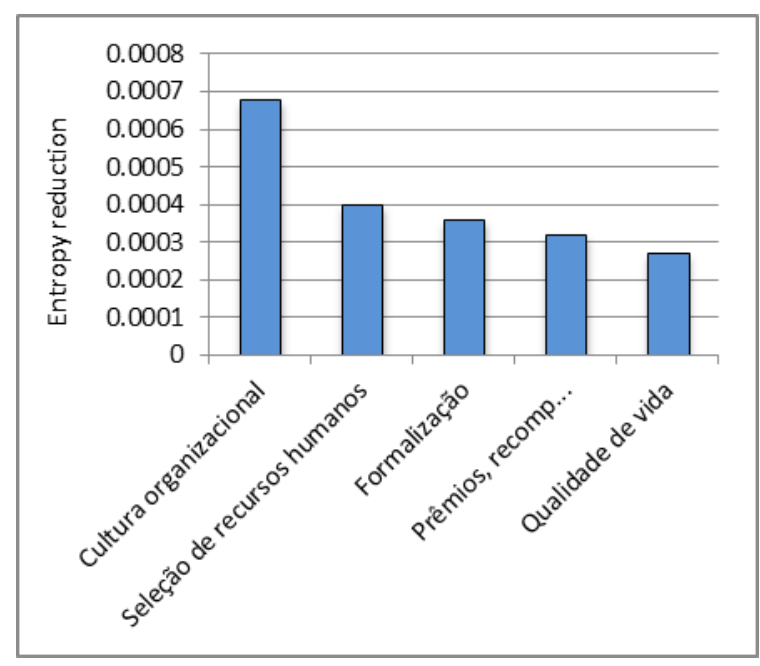

Figura 9 - Resultados da análise de sensibilidade para a subtarefa "requisitar a ação corretiva necessária"

\section{CONCLUSÕES}

Este artigo apresentou a aplicação de uma metodologia para $\mathrm{ACH}$ baseada num modelo genérico de dependência para mapear fatores que impactam no desempenho humano por meio de uma RB. A quantificação da RB foi suportada por valores preprocessados de probabilidade de erro humano, por meio do modelo prospectivo de desempenho humano da TECHR. Este modelo foi aplicado à análise dos fatores que influenciam a probabilidade de erro humano durante manobras de navios em portos. Apesar de não ser ajustado para atender às especificidades de um porto qualquer, o modelo permitiu realizar uma análise preliminar de sensibilidade. Nessa análise, foi possível identificar os principais fatores organizacionais que contribuem para a probabilidade de erro humano em cada ação humana desempenhada. Essas ações humanas foram determinadas pelo acompanhamento em campo de manobras e simulações, organizadas segundo uma análise hierárquica de tarefas.

$\mathrm{O}$ trabalho aqui apresentado reflete as atividades iniciais de um projeto de pesquisa e desenvolvimento para avaliar a contribuição do fator humano ao risco em manobras de navios em portos e hidrovias no Brasil. Até o momento, o modelo permite calcular a probabilidade de erro humano para cada subtarefa individual, além de determinar os principais fatores que as influenciam. Trabalhos futuros incluirão a implementação dos resultados em modelos de acidentes (e.g., árvores de falhas e árvores de eventos) para descobrir o quanto o erro humano impacta no risco total.

Não obstante, como destacado anteriormente, este modelo é genérico e precisa de informações locais para se ajustar a um porto específico. Dessa maneira, ainda no que se refere aos trabalhos futuros, estes incluem a aplicação do modelo completo (já incluindo as sequências de acidentes) para um porto ou hidrovia específicos. O ajuste inclui a opinião de especialistas locais e evidências empíricas, quando disponíveis. Isso permitirá a geração de resultados mais precisos, os quais, inclusive, poderão ser comparados com o próprio modelo genérico.

\section{REFERÊNCIAS BIBLIOGRÁFICAS}

[1] ABREU, D. T. M. P.; MATURANA, M. C.; MARTINS, M. R.; SCHENK JR., S.R. Human Reliability Analysis of Ship Maneuvers in Harbor Areas. In: ASME $201938^{\text {th }}$ International Conference on Ocean, Offshore \& Arctic Engineering (OMAE 2019). Proceedings of the ASME 2019 38 $^{\text {th }}$ International Conference on Ocean, Offshore \& Arctic Engineering. Glasgow, ASME, 2019. p. 1-11. No prelo.

[2] AL-SHAMMARI, N. F. S. H.; OH, J. Effects of Human Error On Marine Safety: 
Case Study. Journal of Enginering Research and Applications. Ijera, v. 8, Issue 11 (Part II), p. 11-19, 2018.

[3] INTERNATIONAL MARITIME ORGANIZATION. Guidelines for Formal Safety Assessment (FSA) for Use in the IMO Rule-Making Process. London: IMO, 2002.

[4] MARTINS, M.R.; MATURANA, M.C. Human Error Contribution in Collision and Grounding of Oil Tankers. Risk Analysis. Wiley, v. 30, n. 4, p. 674-698, 2010.

[5] SWAIN, A.; GUTTMAN, H. Handbook of Human Reliability Analysis with Emphasis on Nuclear Power Plant Applications. Albuquerque: Sandia National Laboratories, 1983.

[6] MARTINS, M.R.; MATURANA, M.C. Application of Bayesian Belief Networks to the Human Reliability Analysis of an Oil Tanker Operation Focusing on Collision Accidents. Reliability Engineering and Systems Safety. Elsevier, v. 110, p. 89-109, 2013.

[7] SOTIRALIS, P.; VENTIKOS, N. P.; HAMANN, R.; GOLYSHEV, P.; TEIXEIRA, A. P. Incorporation of Human Factors into Ship Collision Risk Models Focusing on Human Centered Design Aspects. Reliability Engineering and Systems Safety. Elsevier, v. 156, p. 210-227, 2016.

[8] HÄNNINEN, M.; KUJALA, P. Influences of variables on ship collision probability in a Bayesian belief network model. Reliability Engineering and Systems Safety. Elsevier, v. 102, p. 27-40, 2012.

[9] MARTIN, J.; MARTIN, S.; PETTIT, S. Container ship size and the implications on port call workload. International Journal of Shipping and Transport Logistics.Inderscience Enterprises, v. 7, n. 5, p. 553-569, 2015.

[10] PEARL, J. Fusion, Propagation, and Structuring in Belief Networks. Artificial Intelligence, Elsevier, v. 29, p. 241-288, 1986.

[11] RUSSEL, S. J.; NORVIG, P. Artificial Intelligence: A Modern Approach. Third Edition. Upper Saddle River: Pearson Education, Inc., 2010.

[12] MATURANA, M. C. Consideração da confiabilidade humana na concepção de sistemas complexos: desenvolvimento da TECHR. 2017. 378 p. Tese (Doutorado em Engenharia Naval e Oceânica) - Escola Politécnica da Universidade de São Paulo, São Paulo, 2017.

[13] BLOOM, B. S.; ENGELHART, M. D.; FURST, E. J.; HILL, W. H.; KRATHWOHL, D. Taxonomy of Educational Objectives: The Classification of Educational Goals by a Committee of College and University Examiners. Handbook 1: Cognitive Domain. New York: David McKay Company, 1956.

[14] REASON, J. Human Error. Cambridge: Cambridge University Press, 1990.

[15] ANNET, J. Hierarchical Task Analysis. In: HOLLNAGEL, E. Handbook of cognitive task design. Mahwah: Lawrence Erlbaum Associates, 2003, cap. 2, p. 17-35.

[16] MARCOT, B. G. Metrics for evaluating performance and uncertainty of Bayesian network models. Ecological Modelling, Elsevier, v. 230, p. 50-62, 2012.

[17] HOSHINO, E.; VAN PUTTEN, I.; GIRSANG, W.; RESOSUDARMO, B. P. YAMAZAKI, S. A Bayesian belief network model for community-based coastal resourcemanagement in the Kei Islands, Indonesia. Ecology and Society. Resilience Alliance, v. 21, n. 2, art. 16, 2016. 